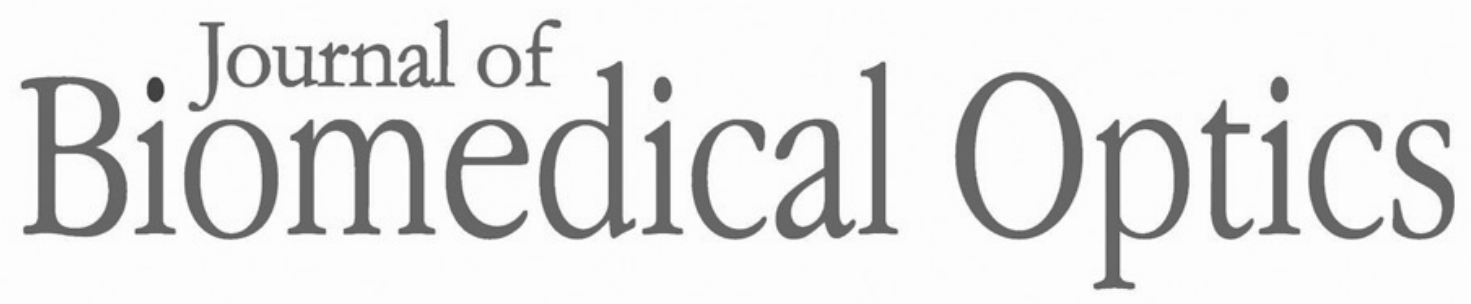

\title{
Catechin reduces phototoxic effects induced by protoporphyrin IX-based photodynamic therapy in the chick embryo chorioallantoic membrane
}

Jaroslava Joniová

Georges Wagnières 


\title{
Catechin reduces phototoxic effects induced by protoporphyrin IX-based photodynamic therapy in the chick embryo chorioallantoic membrane
}

\author{
Jaroslava Joniová* and Georges Wagnières \\ Swiss Federal Institute of Technology (EPFL), Institute of Physics, \\ Laboratory for Functional and Metabolic Imaging, Lausanne, Switzerland
}

\begin{abstract}
Significance: Side effects of many cancer treatments are associated with the production of reactive oxygen species (ROS) in normal tissues. This explains why patients treated by photodynamic therapy (PDT) often suffer from skin photosensitization, whereas those subject to radiotherapies frequently experience damages in various organs, including the skin.

Aim: Catechin, which belongs to the natural flavanols family, is well known for its antioxidant properties. Hence, our main objective was to investigate whether catechin can reduce damages induced by PDT using protoporphyrin IX (PpIX-PDT), an endogenous photosensitizer commonly used in dermatology.

Approach: An in vivo model, the chick embryo chorioallantoic membrane (CAM), was used for this study. An amount of $20 \mu \mathrm{l}$ of a solution containing 5-aminolevulinic acid, a natural precursor of PpIX, was applied topically on the CAM $4 \mathrm{~h}$ before PDTs $\left(405 \mathrm{~nm}, 2.9 \mathrm{~mW} / \mathrm{cm}^{2}\right.$, $\left.1.2 \mathrm{~J} / \mathrm{cm}^{2}\right)$. Catechin was applied at different concentrations $(1$ to $50 \mu \mathrm{M})$ and times $(0$ to $240 \mathrm{~min}$ ) before PDT. In addition, we assessed the potency of catechin to reduce the PpIX fluorescence photobleaching induced by PDT.

Results: We observed that catechin significantly reduces the vascular damages generated by PpIX-PDT. Moreover, we have shown that catechin inhibits PpIX photobleaching.

Conclusions: These observations suggest that catechin significantly reduces the level of ROS produced by PpIX-PDT.

(C) The Authors. Published by SPIE under a Creative Commons Attribution 4.0 Unported License. Distribution or reproduction of this work in whole or in part requires full attribution of the original publication, including its DOI. [DOI: 10.1117/1.JBO.25.6.063807]
\end{abstract}

Keywords: catechin; reactive oxygen species; protoporphyrin IX; photodynamic therapy; phototoxic effect; photoprotection; chorioallantoic membrane; photobleaching.

Paper 190346SSR received Sep. 27, 2019; accepted for publication Dec. 30, 2019; published online Feb. 12, 2020.

\section{Introduction}

Photodynamic therapy (PDT), a minimally invasive and clinically approved procedure, is used for the treatment of different types of (pre)cancers as well as certain forms of age-related macular degeneration. ${ }^{1,2}$ Three key elements are involved in PDT: a nontoxic photosensitizer (PS), light that excites the PS, and molecular oxygen. The PS, after excitation with light, can transfer some of its energy to the latter, which leads to the production of reactive oxygen species (ROS), in particular, singlet oxygen. These ROS induce cell/tissue damages, eventually resulting in cell death by apoptosis, necrosis, and/or autophagy. ${ }^{3-5}$

One of the most extensively used PS for PDT is protoporphyrin IX (PpIX). This fluorescing PS is ubiquitously present and endogenously produced in most living cells as a penultimate step in the biosynthesis of heme. ${ }^{6}$ Its production in certain cancer lesions can be significantly

*Address all correspondence to Jaroslava Joniová, E-mail: jaroslava.joniova@epfl.ch 


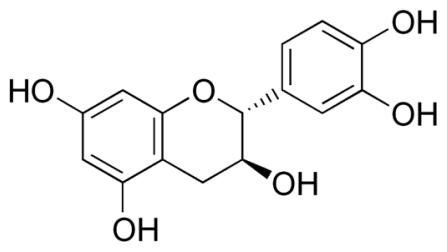

Fig. 1 Chemical structure of catechin.

increased by the administration of some of its approved natural precursors, 5 -aminolevulinic acid (5-ALA), or derivatives thereof. However, normal tissue inflammation, erythema, edema, pain, and/or itching have been reported as side effects of PDT. ${ }^{7}$ One of them results from the skin photosensitizations induced by sunlight when patients are treated with most of the approved PSs. These negative effects, which develop in the course of, or immediately after (hours/days) PDT, are frequently linked to the generation of ROS, especially singlet oxygen, in the normal/healthy tissues. 8 ,9

Therefore, in this study, we have investigated the ability of catechin (Fig. 1), a natural antioxidant belonging to the family of flavonoids (polyphenols), to reduce the phototoxic effects of ROS induced by PpIX-PDT.

Flavonoids are an extensive group of polyphenolic antioxidants naturally occurring in fruits, vegetables, cocoa, chocolate, and several beverages such as wine and green tea. ${ }^{10-12}$ More precisely, catechin belongs to the family of flavanols (or flavan-3-ols), which are able to act as ROS scavengers, especially superoxide anions and singlet oxygen, thus preventing the production of free radicals. ${ }^{13,14}$

Furthermore, catechins also induce indirect antioxidant mechanisms, including the inhibition of pro-oxidant enzymes, the induction of antioxidant enzymes, and many others. ${ }^{15}$

The chicken embryo chorioallantoic membrane (CAM) has been used as an in vivo model in this study to quantify the photoprotective effects of catechin when vascular damages were induced by PpIX-PDT.

The CAM is widely used as a relevant model for studying angiogenic agents due to its developing vascular network. Indeed, the CAM plays the same role as the placenta in mammals, by supplying the nutrition and oxygen to the developing chicken embryo. ${ }^{16}$ During the embryo development, several days after the beginning of the incubation, the CAM is formed with tree-like venules and arterioles that evolve from the initial homogeneous capillary plexus. ${ }^{17}$ Its accessibility and the two-dimensional structure of the vascular network make the CAM an excellent and very convenient model for the visualization of the vascular network and, in particular, to assess the effects of PDT.

In this study, different concentrations of catechin were topically administered at different times before PpIX-PDT.

\section{Material and Methods}

\subsection{Chemicals}

All chemicals were purchased from Sigma-Aldrich, Switzerland.

\section{$2.2 C A M$}

Fertilized chicken eggs were purchased from Animalco AG, Switzerland. For 3 days, eggs were incubated at $37^{\circ} \mathrm{C}$ in a humidified and automatically rotating incubator with the blunt end up. At the embryo development day (EDD) 3, a small hole (diameter: $3 \mathrm{~mm}$ ) was made at the pointed end of the shell and covered with tape. Afterward, eggs were placed back to the incubator in a stationary position until further use. On EDD 12, the hole was enlarged (diameter: $25 \mathrm{~mm}$ ) enabling topical ALA and catechin administration followed by PDT. 


\subsection{PpIX-PDT}

PpIX-based PDT has been described in detail in our previous reports. ${ }^{18}$ Briefly, ALA was dissolved in an aqueous solution of $\mathrm{NaCl}(0.9 \%$ of $\mathrm{NaCl}$ and $20 \mathrm{mg}$ of ALA per ml with a pH of 6.8). An amount of $20 \mu \mathrm{l}$ of this solution was topically applied on the CAM. Then, $4 \mathrm{~h}$ after ALA administration, PDT was conducted with the light delivered by an epifluorescent microscope (Eclipse E 600 FN Nikon) utilizing a 4× objective (Nikon, NA: 0.13, Plan Fluor, WD 17.1). PpIX produced endogenously was excited with light at $405 \mathrm{~nm}$ (filter cube BV-2A, Nikon, Japan). PpIX-PDT was performed with a light dose of $1.2 \mathrm{~J} / \mathrm{cm}^{2}$ (irradiance: $2.9 \mathrm{~mW} / \mathrm{cm}^{2}$ ). This light dose typically induces a grade 4 vascular damage according to the PDT effects scale defined by Lange et al., ${ }^{19}$ i.e., vessels with a diameter $<70 \mu \mathrm{m}$ are all closed by PDT.

\subsection{Catechin Administration}

A stock solution of catechin was dissolved in $\mathrm{EtOH}$ before further dilution in $\mathrm{NaCl}$ (used for the topical administration on the CAM). Different concentrations of catechin $(1,5,10,20,30$, and $50 \mu \mathrm{M}$ ) were applied topically (in $100 \mu \mathrm{l}$ of $\mathrm{NaCl}$ ) to the CAM at different time intervals ranging between 0 and $4 \mathrm{~h}$ prior to PpIX-PDT. In all experiments, the content of EtOH was $<1 \%$. It should be noted that concentrations of catechin are relative to the weight of the embryo at given EDD, i.e., $5 \mathrm{~g}$ at EDD 12. For all control eggs, $100 \mu \mathrm{l}$ of $\mathrm{NaCl}$ (without catechin) was topically applied on the CAM.

\subsection{Angiograms Acquisition and Analysis}

Fluorescent angiograms of the CAM's vascular network were recorded $24 \mathrm{~h}$ after PDT. Angiograms were obtained by an intravenous injection $(20 \mu \mathrm{l})$ of fluorescein isothiocyanatedextran $(25 \mathrm{kD}, 25 \mathrm{mg} / \mathrm{ml})$ dissolved in $\mathrm{NaCl}$. To further improve the contrast between the extravascular space and blood vessels of the CAM, India ink (Parker) was concurrently injected $(100 \mu \mathrm{l})$ under the CAM. It has been proven that India ink, which is also used as a marker for tumor margination in the human skin, is not toxic to the CAMs in our conditions. ${ }^{20}$ Angiograms were acquired with an epifluorescent microscope (Eclipse E 600 FN Nikon) utilizing a $4 \times$ objective (Nikon, NA: 0.13, Plan Fluor, WD 17.1). PDT potency along with the assessment of the catechin photoprotection was quantified by the quantitative analysis of these fluorescent angiograms using an ImageJ Macro (NIH, Bethesda, Maryland) developed in our laboratory. ${ }^{20}$

\subsection{PpIX Photobleaching Study}

Fluorescent images for this substudy have been obtained with the same microscope setup as described above. A BV-2A filter cube (excitation: $405 \mathrm{~nm}$; Nikon, Japan) combined with a longpass emission filter transmitting light above $610 \mathrm{~nm}$ (E610LP filter; Chroma, Irvine, California) was used for PpIX fluorescence visualization and relative quantification. Images were recorded at the beginning (first $5 \mathrm{~s}$ ) and at the end (last $5 \mathrm{~s}$ ) of the PDT irradiations. The ImageJ software was then used to quantify the PpIX fluorescence intensity changes (at the beginning and at the end of illumination) in the presence and absence of catechin. This study was carried out with a light dose of $10 \mathrm{~J} / \mathrm{cm}^{2}$ at $405 \mathrm{~nm}$, and $10 \mu \mathrm{M}$ catechin was administered $15 \mathrm{~min}$ before PDT.

\section{Results and Discussion}

\subsection{Study of the Photoprotective Effects of Catechin Applied at Different Concentrations}

As mentioned above, PpIX is one of the most frequently used PSs for PDT. Its phototoxic effects are well documented, in particular, those induced in the vascularization. ${ }^{18,21-23}$ Hence, in this study, it has been used as PS for the induction of vascular damages in the CAM. On the basis of a preliminary study, we established the spectral and radiometric conditions inducing grade 4 vascular damages according to Lange et al. ${ }^{19}$ (data not shown). We concluded that light at 
$405 \mathrm{~nm}$ applied with a dose of $1.2 \mathrm{~J} / \mathrm{cm}^{2}$ and an irradiance of $2.9 \mathrm{~mW} / \mathrm{cm}^{2}$ was appropriate. These conditions were used in all experiments presented in Secs. 3.1 and 3.2.

In the first step, we studied the photoprotective effects of catechin applied topically at different concentrations 15 min before PDT (1 to $50 \mu \mathrm{M}$ ). Figure 2(a) shows illustrative fluorescence angiograms acquired $24 \mathrm{~h}$ after PDT. A quantitative analysis of fluorescence angiograms [Fig. 2(b)] to assess the efficacy of PpIX-PDT, i.e., to determine the diameter of the smallest vessels that did not close after PpIX-PDT, showed that, in the control samples, this diameter was in the order of $100 \mu \mathrm{m}$. On the other hand, PpIX-PDT was significantly less potent at all concentrations of catechin. More precisely, the small concentrations (1 and $5 \mu \mathrm{M})$ reduced the PpIXPDT effects in such a way that the smallest vessels that did not close had an average diameter of about $45 \mu \mathrm{m}$. Similarly, the administration of catechin at higher concentrations $(20,30$, and
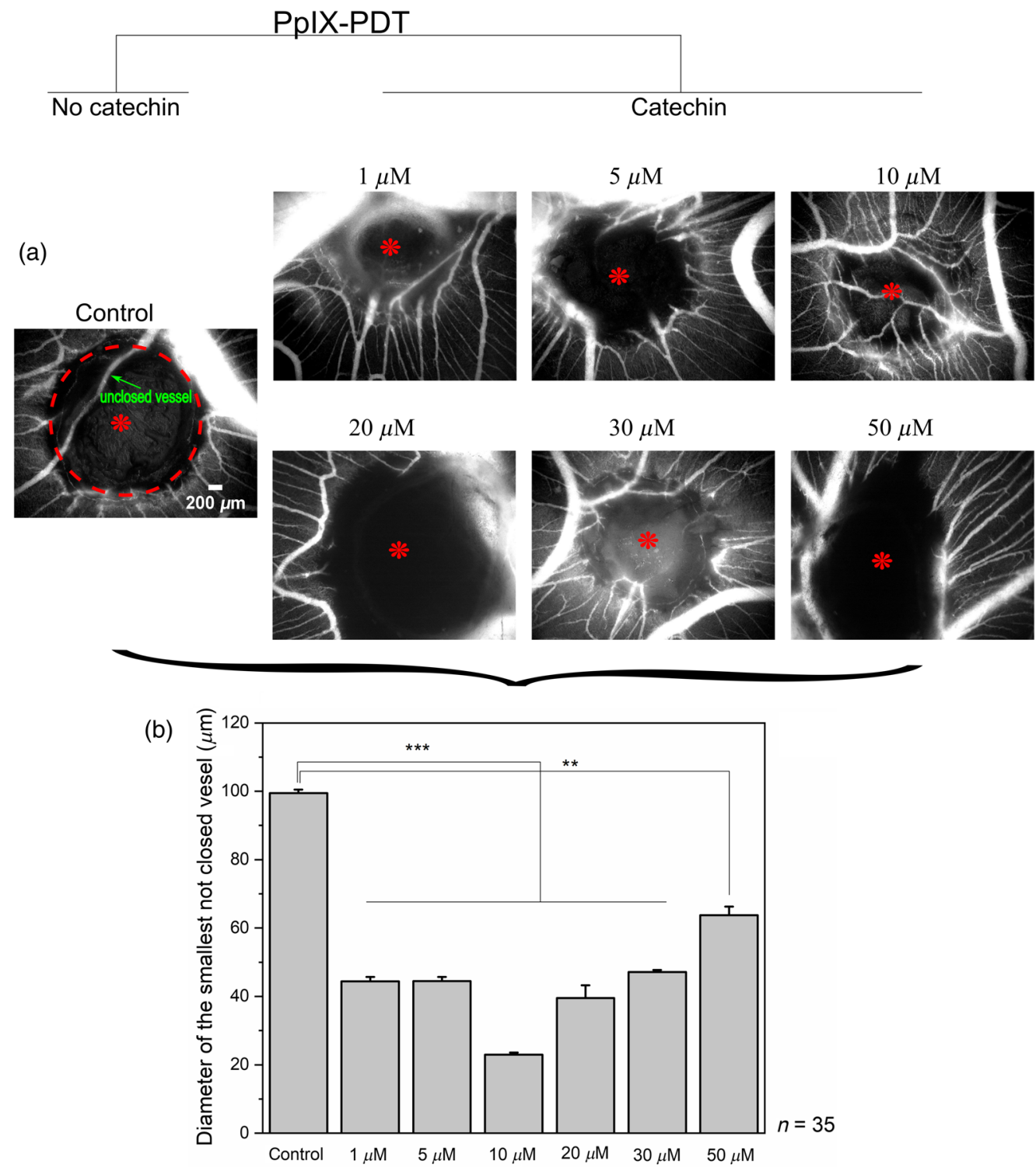

Fig. 2 Assessment of PpIX-PDT on the CAM in the presence of different concentrations of catechin. (a) Representative FITC-dextran fluorescent angiographies of the CAM in control samples (no catechin) and in the presence of different concentrations of catechin. Images were acquired $24 \mathrm{~h}$ after PDT. All images have the same magnification. A typical unclosed vessel as well as the illuminated circular area is presented in the control image only for the sake of clarity. Red asterisks in the images represent the centers of the illumination with a diameter of $2 \mathrm{~mm}$. (b) Quantitative analysis of the fluorescent images. Number of eggs: $n=35$. The error bars represent the standard error of the mean. Significant $p$ values are represented as ${ }^{\star *} p \leq 0.01,{ }^{* \star *} p<0.001$. 
$50 \mu \mathrm{M}$ ) led to a reduction of the smallest not closed vessel down to 39,47 , and $64 \mu \mathrm{m}$, respectively. An amount of $10 \mu \mathrm{M}$ of catechin showed the most significant photoprotective effect since the average diameter of the smallest unclosed vessels was about $23 \mu \mathrm{m}$. It should be noted that "no light" controls (in the presence of catechin only or a combination of catechin with ALA) were conducted, resulting in an absence of vascular changes (data not shown).

Although the family of catechins is well recognized for its antioxidant properties, it should be noted that they are also able to act as pro-oxidants generating ROS. ${ }^{24,25}$ Indeed, although their antioxidant properties are due to their ability to act as free radical/ROS scavengers, their presence can stimulate hemoglobin-induced protein oxidation. This could be caused by their antioxidant capability, which promptly induces an oxidative degradation of hemoglobin, ${ }^{22}$ an effect that may explain the concentration-dependent photoprotective properties of catechin.

\subsection{Determination of the Optimal Catechin Administration Time Before PpIX-PDT}

Following the catechin concentration study reported in Sec. 3.1, we investigated the influence of the role played by the time separating the catechin administration and PpIX-PDT. Since $10 \mu \mathrm{M}$ of catechin was found to be optimal, this concentration was used for the second substudy. Figure 3 shows the vascular effects of PpIX-PDT when catechin was applied topically at different times before illumination on the CAM.

We observed that the presence of $10-\mu \mathrm{M}$ catechin reduces the PpIX-PDT effect at all times. When administered just (i.e., $<30 \mathrm{~s}$ ) before PpIX-PDT illumination, the smallest vessel that did not close was in the order of $51 \mu \mathrm{m}$. Increasing the time between catechin administration and illumination up to about 15 min led to more potent inhibitions of the vascular effects of PpIXPDT. Indeed, when catechin was applied 7 min before PDT, the average diameter of the smallest unclosed vessel was $48 \mu \mathrm{m}$, whereas this diameter went down to $23 \mu \mathrm{m}$ at the optimal time of $15 \mathrm{~min}$. With longer times (30 min and more), catechin became less and less potent to generate a photoprotection.

According to our knowledge, there is no literature on the catechin pharmacokinetics in the CAM. However, studies conducted on rodents, dogs, rabbits, and humans showed that, after oral administration, catechin is mainly metabolized by phase- 2 conjugation process in the liver and intestine ${ }^{26}$ In our study conducted on the CAM, the reduced photoprotection observed at times $>30$ min could be explained by the catechin internalization by the CAM and chicken embryo itself.

\subsection{Assessment of the ROS Production During PDT Based on the Measurement of the PpIX Fluorescence Photobleaching}

Measurements conducted in this part of the study were based on the observation that PpIX photobleaches during PDT, an effect that is directly related to the ROS (mostly singlet oxygen) production. ${ }^{27}$ Indeed, Moan and Berg ${ }^{28}$ demonstrated that the more ROS are created, the more PpIX is photobleached. Therefore, it is of interest to determine if a correlation exists between the photoprotective effects of catechin and the PpIX photobleaching. Thus, this estimation of the protective effect of catechin was compared with its ability to prevent photobleaching of PpIX. Figure 4 shows the ratio of the fluorescence intensity of PpIX measured at the beginning (first $5 \mathrm{~s}$ ) divided by this intensity measured at the end of PDT (last $5 \mathrm{~s}$ ), both for a control group (no catechin) and in the presence of $10-\mu \mathrm{M}$ catechin applied 15 min before PpIX-PDT. If no photobleaching would occur, the ratio of the PpIX fluorescence at the beginning and at the end of illumination would be equal to 1 .

For the purpose of acquiring a good signal-to-noise ratio, the light dose used for this part of the study was increased to $10 \mathrm{~J} / \mathrm{cm}^{2}$ for all conditions (controls and $10-\mu \mathrm{M}$ catechin). It should be noted that increasing the light dose did not preclude the photoprotective effect of catechin. At the same time, it is interesting to point out that when photobleaching experiments of PpIX were conducted with $1.2 \mathrm{~J} / \mathrm{cm}^{2}$ (see Secs. 3.1 and 3.2), the PpIX photobleaching was almost not measurable (data not showed), although the tissue damages were significant. On the whole, these observations suggest that only a small fraction of the PpIX present on the CAM membrane $4 \mathrm{~h}$ 


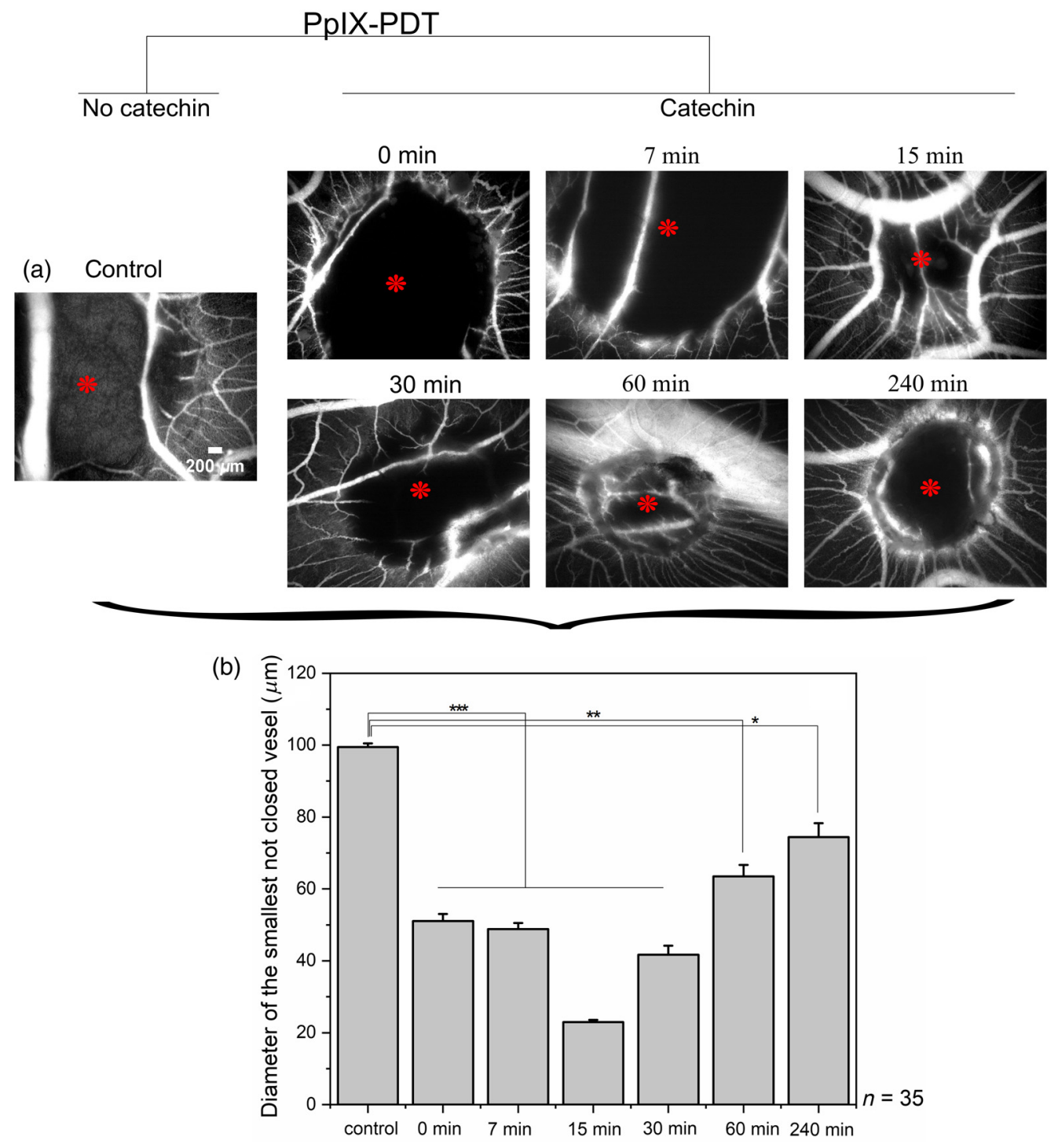

Fig. 3 Effects of PpIX-PDT on the CAM when catechin is applied at different times before illumination. (a) Representative FITC-dextran fluorescent angiographies of the CAM in control samples (no catechin) and in the presence of $10 \mu \mathrm{M}$ of catechin administered at different times before PpIXPDT. Images were acquired $24 \mathrm{~h}$ after PDT. Red asterisks in the images represent the center of the illumination spots with a diameter of $2 \mathrm{~mm}$. (b) Quantitative analysis of the fluorescent images for different times between catechin administration and PpIX-PDT. Number of eggs: $n=35$. The error bars represent the standard error of the mean. Significant $p$ values are represented as ${ }^{*} p \leq 0.05,{ }^{* *} p \leq 0.01,{ }^{* \star *} p<0.001$.

after ALA application is actually localized in compartment(s), probably the mitochondria, playing a crucial role in the photosensitization process. This suggests that the majority of the PpIX fluorescing in the CAM in our conditions plays a reduced role in the photosensitization process, if any, probably because it is outside these critical compartments.

Nevertheless, as shown in Fig. 4, 10- $\mu \mathrm{M}$ catechin not only reduces the PpIX photobleaching, but leads to an increase of the PpIX fluorescence in the CAM. This effect is probably due, at least in part, to the ability of catechin to reduce the ROS concentration. It could also be that the increase of PpIX fluorescence observed during PDT in the presence of catechin is due to a chelation of iron by this flavanol, as postulated by Samman et al. ${ }^{29}$ This mechanism may impair the availability of iron to convert PpIX in heme, thus leading to an increase of the PpIX fluorescence detected in the CAM. 


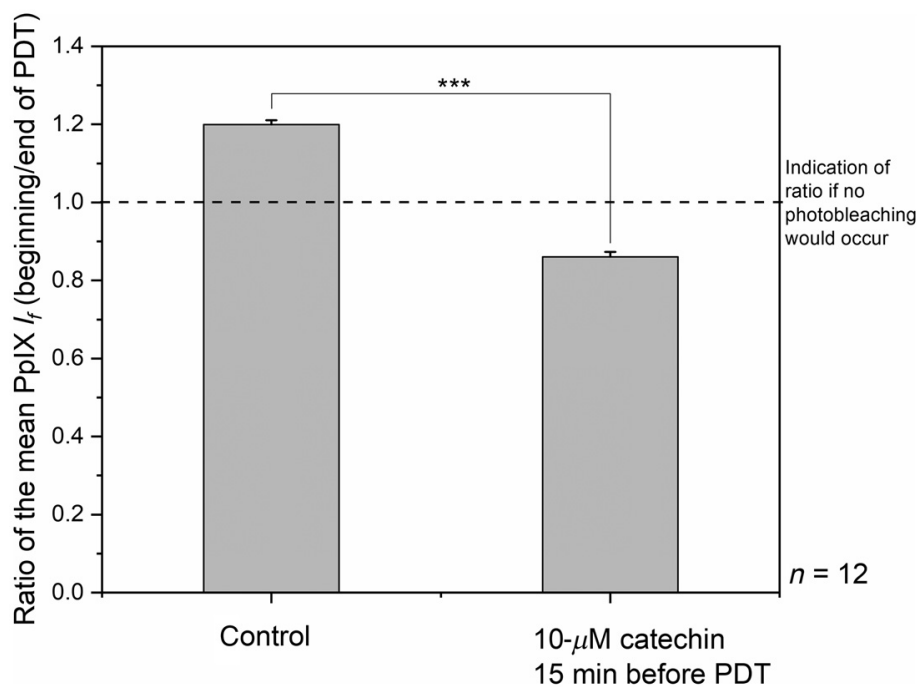

Fig. 4 Reduction of the PpIX photobleaching with catechin. PplX fluorescence intensity was recorded in the first $5 \mathrm{~s}$ and last $5 \mathrm{~s}$ of the PDT illumination. These values were divided and compared for the control samples and when 10- $\mu \mathrm{M}$ catechin was administered 15 min before PDT. Number of studied eggs: $n=12$. The error bars represent the standard error of the mean. Significant $p$ value is represented as ${ }^{* \star *} p<0.001$.

\section{Conclusion}

It is well known that patients treated by PDT often suffer from skin photosensitization. In addition, those patients subject to radiotherapies frequently experience damages in various organs, including the skin, as a consequence of the ROS production in the tissues. Since the family of catechins is well known for its antioxidant properties, we investigated if its topical application could induce photoprotective effects in normal tissues during PpIX-PDT and/or if it could be used to protect the skin of patients against photosensitization induced by PpIX-PDT. The influence of the catechin concentration and the time between its administration and PpIX-PDT were studied on the CAM vascularization. We observed that catechin reduced the PpIX-PDT potency in all conditions we explored, whereby $10-\mu \mathrm{M}$ catechin administered topically 15 min before PDT had the most potent photoprotective effect. In addition, we demonstrated that, in these conditions, catechin diminishes the PpIX photobleaching induced by PpIX-PDT. Although additional experiments exploring other administration conditions carried out on different model systems are necessary, our results suggest that catechin is an interesting candidate to protect normal tissues during PDT or radiotherapy.

\section{Disclosure}

Authors have no conflicts of interest to declare.

\section{Acknowledgments}

The authors are grateful for the financial support of AC BioScience and the Swiss National Science Foundation (project 315230_185262/1).

\section{References}

1. H. van den Bergh, "Photodynamic therapy of age-related macular degeneration: history and principles," Semin. Ophthalmol. 16(4), 181-200 (2001).

2. H. van den Bergh, J.-P. Ballini, and M. Sickenberg, "On the selectivity of photodynamic therapy of choroidal neovascularization associated with age-related macular degeneration," J. Fr. Ophtalmol. 27(1), 75-78 (2004). 
3. C. Fleury, B. Mignotte, and J.-L. Vayssière, "Mitochondrial reactive oxygen species in cell death signaling," Biochimie 84(2-3), 131-141 (2002).

4. M. Redza-Dutordoir and D. A. Averill-Bates, "Activation of apoptosis signalling pathways by reactive oxygen species," Biochim. Biophys. Acta, Mol. Cell Res. 1863(12), 2977-2992 (2016).

5. M. B. Azad, Y. Chen, and S. B. Gibson, "Regulation of autophagy by reactive oxygen species (ROS): implications for cancer progression and treatment," Antioxid. Redox. Signal. 11(4), 777-790 (2009).

6. M. Sachar, K. E. Anderson, and X. Ma, "Protoporphyrin IX: the good, the bad, and the ugly," J. Pharmacol. Exp. Ther. 356(2), 267-275 (2016).

7. F. Borgia et al., "Early and late onset side effects of photodynamic therapy," Biomedicines 6(1), 12 (2018).

8. M. C. DeRosa and R. J. Crutchley, "Photosensitized singlet oxygen and its applications," Coord. Chem. Rev. 233-234, 351-371 (2002).

9. J. M. Dąbrowski, "Reactive oxygen species in photodynamic therapy: mechanisms of their generation and potentiation," Chapter 9 in Advances in Inorganic Chemistry, R. van Eldik and C. D. Hubbard, Eds., Vol. 70, pp. 343-394, Academic Press (2017).

10. M. G. Hertog et al., "Dietary antioxidant flavonoids and risk of coronary heart disease: the Zutphen Elderly Study," Lancet 342(8878), 1007-1011 (1993).

11. J. Kühnau, "The flavonoids. A class of semi-essential food components: their role in human nutrition," World Rev. Nutr. Diet. 24, 117-191 (1976).

12. J. Allgrove and G. Davison, "Dark chocolate/cocoa polyphenols and oxidative stress," Chapter 19 in Polyphenols in Human Health and Disease, R. R. Watson, V. R. Preedy, and S. Zibadi, Eds., pp. 241-251, Academic Press, San Diego (2014).

13. S. Rafat Husain, J. Cillard, and P. Cillard, "Hydroxyl radical scavenging activity of flavonoids," Phytochemistry 26(9), 2489-2491 (1987).

14. C. G. Fraga et al., "Basic biochemical mechanisms behind the health benefits of polyphenols," Mol. Aspects Med. 31(6), 435-445 (2010).

15. J. Bernatoniene and D. M. Kopustinskiene, "The role of catechins in cellular responses to oxidative stress," Molecules 23(4), 965 (2018).

16. V. G. Djonov, H. Kurz, and P. H. Burri, "Optimality in the developing vascular system: branching remodeling by means of intussusception as an efficient adaptation mechanism," Dev. Dyn. 224(4), 391-402 (2002).

17. A. F. Smith et al., "Microvascular hemodynamics in the chick chorioallantoic membrane," Microcirculation 23(7), 512-522 (2016).

18. F. Piffaretti et al., "Real-time, in vivo measurement of tissular $\mathrm{pO}_{2}$ through the delayed fluorescence of endogenous protoporphyrin IX during photodynamic therapy," J. Biomed. Opt. 17(11), 115007 (2012).

19. N. Lange et al., "A new drug-screening procedure for photosensitizing agents used in photodynamic therapy for CNV," Invest. Ophthalmol. Vis. Sci. 42(1), 38-46 (2001).

20. P. Nowak-Sliwinska et al., "Processing of fluorescence angiograms for the quantification of vascular effects induced by anti-angiogenic agents in the CAM model," Microvasc. Res. 79(1), 21-28 (2010).

21. C. J. Fisher et al., "ALA-PpIX mediated photodynamic therapy of malignant gliomas augmented by hypothermia," PLoS One 12(7), e0181654 (2017).

22. M. B. Ericson, A.-M. Wennberg, and O. Larkö, "Review of photodynamic therapy in actinic keratosis and basal cell carcinoma," Ther. Clin. Risk Manage. 4(1), 1-9 (2008).

23. M. Goldman and D. Atkin, "ALA/PDT in the treatment of actinic keratosis: spot versus confluent therapy," J. Cosmet. Laser Ther. 5, 107-110 (2003).

24. C. S. Yang et al., "Effects of tea catechins on cancer signaling pathways," Enzymes 36, 195-221 (2014).

25. J. D. Lambert and R. J. Elias, "The antioxidant and pro-oxidant activities of green tea polyphenols: a role in cancer prevention," Arch. Biochem. Biophys. 501, 65-72 (2010).

26. K. O. Chu and C. C. P. Pang, "Pharmacokinetics and disposition of green tea catechins," in Pharmacokinetics and Adverse Effects of Drugs: Mechanisms and Risks Factors, N. Malangu (2018). 
Joniová and Wagnières: Catechin reduces phototoxic effects induced by protoporphyrin IX-based...

27. G. S. Cox, C. Bobillier, and D. G. Whitten, "Photooxidation and singlet oxygen sensitization by protoporphyrin IX and its photooxidation products," Photochem. Photobiol. 36(4), 401-407 (1982).

28. J. Moan and K. Berg, "The photodegradation of porphyrins in cells can be used to estimate the lifetime of singlet oxygen," Photochem. Photobiol. 53(4), 549-553 (1991).

29. S. Samman et al., "Green tea or rosemary extract added to foods reduces nonheme-iron absorption,” Am. J. Clin. Nutr. 73(3), 607-612 (2001).

Biographies of the authors are not available. 